pneumonia in cattle and mastitis in dairy cattle was continued. The Division has also continued its studies in fleece chemistry, yield and fibre measure. ment and on the sheep blowfly problem, and an investigation on sheep dips was initiated during the year.

In addition to continuing its various soil surveys, the Division of Soils has been concerned with the use of a spectrograph and the polarograph for the determination of traces of metals in soils and in plant and animal diseases.

The work of the Division of Forest Products has continued to expand during the year and, in addition to the chemistry of wood and pulp and paper investi. gations, has been concerned with the preservation of timber, the seasoning of timber and various problems in the wood manufacturing industry.

The Division for Food Preservation and Transport occupied its new laboratory at Homebush Bay, Sydney, during the year and has completed its work on the cooling of beef in sides. Other work has dealt with the loss of bloom on chilled beef, the storage of oranges, peaches, plums, pears and grapes as well as on apples and eggs. Further progress has been made during the year by the Council in the provision of staff and facilities for fishery investigations, in which aerial reconnaissance has again been used.

\title{
The Dutch Congress of Natural Science and Medicine
}

$\mathrm{T}$ HE twenty-seventh Dutch Congress of Natural Science and Medicine was held at Nimeguen, Holland, during April 11-13, under the presidency of Prof. G. Holst. Reference was made to the general lectures in the programme in a note in NATURE of April 1, p. 554. At a joint meeting of the physical and chemical sections Prof. Errera, of Brussels, gave a special address to the Congress on his investigations of the absorption of organic compounds in the infra-red part of the spectrum. Besides the wellknown absorption bands belonging to special atomic configurations, new absorption bands appear which must be ascribed to multi-molecules (association). In solutions, there appear special bands due to the interaction of an atomic group of the solvent with the molecule in solution.

\section{Mathematical Physics and Physics}

Among the papers presented to the section on mathematical physics, the following may be mentioned. Methods of approximation of the general wave equation were discussed with special reference to the Schrödinger equation. Prof. de Laer-Kronig treated a new perturbation theory, leading to a difference equation, which can be solved numerically by the application of an extension of the well-known Wentzel-Kramers-Brillouin approximation. It was shown by Prof. Zernike that the intensity of light in the vicinity of a focus can be described, even for the most general boundary conditions, by a special kind of orthogonal functions, introduced by him. Dr. Bremmer demonstrated that the WentzelKramers-Brillouin approximation of the wave equation may be considered as the first of a series of terms, which can all be interpreted physically. Ehrenfest's theorem of the classical motion of the centre of a wave packet describing a particle in quantum-mechanics was extended by Prof. Fokker for a very general type of propagation equation.

In the physical section, Dr. Matthijs gave an account of measurements on the thermo-electric force of superconducting metals in the neighbourhood of the transition point, showing that superconductivity becomes evident at a temperature superior to the transition point.

In a discussion on nuclear physics, Mr. Barendregt reported experiments on pair formation by $\beta$-rays, which process appears to take place much more frequently than theory predicts. Dr. Heyn gave an account of experiments, made in collaboration with Mr. Aten, jun., and Dr. Bakker, on the fission of the uranium and the thorium nuclei by neutron bombardment. From experimental evidence it follows that these nuclei can break up in more than one way.

\section{Chemistry}

In the chemical section, Mr. van Pelt communicated experiments on the bromination of pyridine and compounds containing a pyridine ring. It is found that, depending on the temperature, either ortho- or meta-substitution occurs. Dr. Heertjes dealt with the measurement of the density of fibres. A gas-volumetric method with helium was used. Evidence was produced for the assumption that water is absorbed by cotton in two different ways. Prof. Aten described an objective colorimeter which can be constructed at very low cost using ordinary laboratory apparatus. Mr. Troelstra discussed the latest improvements in electrodialysis.

\section{BIOLOGY}

The programme of the biological section contained a large variety of communications. The main topic was the need of magnesium in plants (O. de Vries) and animals (Sjollema). Further botanical subjects dealt with were the transport of substances (Arisz), the relation between yield and the ratio between roots and sprouts (Boonstra), bacterial leaf-symbionts (De Jongh), antagonisms between micro-organisms (Westerdijk), the influence of colchicine on nuclear divisions (Wellensiek) and problems in the rooting of cuttings (Funke, Hubert and Rappaport).

Zoological subjects discussed included: Colorado beetle (Roepke), the life of termites in the Netherlands Indies (Kalshoven), metabolism in trypanosomes as a basis for control (Krijgsman) and the power of orientation of migratory birds (Van Dobben).

In addition, pollen analysis investigations (Florschütz), vertebrate fauna (van der Vlerk) and botanical investigations (Polak) of the quaternary in the Netherlands were discussed in the geological section, while the general lectures of Ornstein on physics and biology and of Gorter on very thin membranes attracted considerable attention from biologists. 